\title{
IL Y A 150 ANS
}

\section{Les arbres Marie-Louise et du Roi de Rome}

On a pu récemment lire dans cette Revue ce que furent “ les arbres de la liberté en l'an II ) (*).

Ce n'était pas la première fois en 1790 - ni la dernière - que les hommes plantaient solennellement des arbres destinés à maintenir le souvenir de quelque événement politique ou militaire important. On trouvait encore à Banassac (Lozère) en 1911, un tilleul planté sous François $I^{\text {er }}$ pour fêter la victoire de Marignan (Revue des Eaux et Forêts, 1911, page 477).

Mais l'un des plus vastes mouvements connus de plantation d'arbres ( historiques ) eut pour cadre le Premier Empire: il s'agissait de maintenir le souvenir du mariage de Napoléon $I^{\text {er }}$ et de Marie-Louise d'Autriche, et surtout peu après, de la naissance du (" roi de Rome ).

Une intéressante étude en a été faite dans la Revue des Eaux et Forêts de 1911, par P. Weyd (pages 449-461).

L'initiative vint en $1810 \mathrm{du}$ maire d'une petite commune des environs de Liége, Schleiden: on sait qu'alors la France débordait largement de ses frontières actuelles!

Le " Moniteur ) du 8 juin 1810 écrivait:

“ M. Begasse, maire de Schleiden, a célébré l'époque mémorable " du mariage de Sa Majesté l'Empereur en faisant exécuter une très " belle plantation consistant en 222 arbres... sur un terrain commu" nal qui depuis a reçu le nom de place Napoléon... On ne peut " qu'applaudir à l'idée heureuse qu'a eue M. BEGASSE, qui a été " parfaitement secondé par ses administrés et les gardes fores( tiers ).

(*) Revue Forestière Française, novembre 1961, p. 685-692 et février 1962 (les lecteurs parlent). 
Le Directeur Général des Eaux et Forêts ne manqua pas d'officialiser et de patronner une aussi louable pratique par le canal de deux circulaires administratives:

- la circulaire no 416 du 4 juin 1810 pour les « arbres MarieLouise ",

- la circulaire no 434 du 23 mars 1811, pour les “ arbres du roi de Rome ).

- Cette dernière s'exprimait ainsi:

" J'ai beaucoup applaudi au zèle avec lequel les préposés ont con" sacré par des plantations la mémoire de l'auguste mariage de LL.

( $M M . I I$. et RR.

"Un événement non moins fortuné doit exciter le même élan: il " faut que dans tous les triages, des arbres contemporains de la nais" sance du Roi de Rome prouvent un jour que, jusqu'au fond même " des forêts, tout tressaillit de joie à son avènement au monde ").

L'affaire prit vite un grand développement: les Annales forestières de 1810 et 1811 rendent compte du plus grand nombre des plantations effectuées dans toutes les conservations de France.

Nous prendrons comme modèle celle qui fut réalisée en forêt du Boscodon, près de Gap, sur le plateau de la Faurrie. Trente arbres - chênes, ormes et tilleuls - furent plantés en un cercle de 60 mètres de circonférence. Dans le milieu du cercle, on disposa deux chênes, l'un en l'honneur de l'Empereur, l'autre en l'honneur de l'Impératrice.

Le sous-préfet d'Embrun, le maire de la ville, tout le personnel forestier de la conservation étaient là.

La fête commença par des salves de mousqueterie tirées par les gardes. Puis l'inspecteur local (M. Goutard) prononça un discours lyrique et émouvant bien dans le style quaimaient nos ancêtres il y a cent cinquante ans:

\section{( Messieurs,}

"Qu'elle est touchante, combien elle ćmeut doucement le cour, " la circonstance qui nous rassemble aujourd'hui! Ce n'est point " l'adulation qui commande ces hommages; c'est le respect, c'est ". l'amour filial qui les inspire, c'est le sentiment qui les rend, en " effet. Messieurs, n'est-ce pas une fête de famille, n'est-ce pas " un jour d'allégresse pour tous les Français que celui oì leur sou" verain, leur père, sans cesse occupé de leur bonheur, unit à son " sort la plus vertueuse comme la plus illustre des princesses? C'est " pour consacrer le souvenir de ce glorieux hymen que nous plan" tons ces chênes, dont la tête altière se perdant dans les nues rap- 
" pellera aux races futures, non pas la mémoire de ces monarques " chéris (car elle est immortelle), mais bien l'attachement sans bor" nes et la reconnaissance inexprimable de leurs fidèles sujets; oui, " couple auguste et généreux, vous avez encore un trône dans nos " cours, vos vertus l'y ont élevé, notre amour et notre admiration " pour vos personnes sacrées en garantissent la durée à jamais. ") etc..., etc...!

Après ce discours plein d'émotion, on planta les arbres solennellement, et il ne resta plus, bien entendu, qu'à se rendre dans une salle où un banquet offert par l'inspecteur attendait tout le monde.

$\mathrm{Au}$ dessert, après un toast en l'honneur de leurs majestés, les cris répétés de “Vive l'Empereur! Vive l'Impératrice! ) se confondirent avec de nouvelles salves de mousqueterie effectuées par les. gardes.

Des scènes semblables se répétèrent aux quatre coins de France, et bien sûr aussi dans les conservations de Koblentz (25 637 arbres plantés), Bruxelles (31 775 arbres), Alexandrie (en Italie, 1114 arbres et de nombreux semis), etc...

On peut noter deux types de " variantes », bien faites pour éclairer d'un jour différent la personnalité des hauts fonctionnaires locaux.

Certains voulurent ajouter leur plantation votive “ privée » à l'acte officiel. Citons tel conservateur d'Orléans qui, " pour exemple à ses subordonnés ", planta lui-même à titre personnel 40 arbres commémoratifs sur un terrain environné de fossés de défense.

D'autres représentants de l'Empereur surent au contraire placer l'intérêt général au-dessus de la manifestation de loyalisme. C'est ainsi que le préfet des Alpes-Maritimes félicita, en ces termes l'inspecteur des forêts de Nice qui lui rendait compte des " arbres du roi de Rome ) nombreux et formant massif plantés dans sa circonscription :

" J'applaudis à cette opération avec d'autant plus de satisfaction " que c'est un commencement de régénération très important pour "le département que j’ai souvent désiré, ct qui sera, je l’espère, " soutcnu et suivi avec zèle et persévérance... Je recevrai volontiers, " toutes les années à pareille époque, un état de la situation des " plantations et semis, avec la nature de leurs progrès, et en même " tomps que celui des nouvelles opérations qui y seront ajoutées. "

Voilà certes un bon administrateur, intelligent et avisé!

Vint la campagne de Russie, la Restauration... Qu'allait-il se passer? Le nouveau régime sut agir avec sagesse et modération. 
Voici le texte de la circulaire $n^{\circ} 569$ du 8 mai 1816:

" Il est peu d'arrondissements forestiers, Monsieur, oì il n'ait " été planté des arbres à l'époque de la naissance du fils de Buona" parte; si parmi ces arbres il s'en trouve quelques-uns qui portent " des empreintes emblématiques, vous aurez soin de faire disparâे" tre ces empreintes, et si cela ne peut avoir lieu sans dommage pour "les arbres, vous les mettrez en vente dans la forme ordinaire.

" Je vous prie de donner à cet égard les instructions nécessaires " aux agens qui vous sont subordonnés, et de me rendre compite ( de ce qui aura été fait ).

Il n'y eut donc aucune destruction. Mais une partie des arbres plantés avait péri dès la sécheresse de l'été 1811 .

D'autres, plus heureux, vivent encore de nos jours. En voici un exemple sûr.

Etant il y a dix ans Ingénieur forestier à Dijon, nous nous souvenons très bien que le Chef de District des Eaux et Forêts à Longchamp (entre Dijon et Auxonne), nous montra, dans la parcelle 70 de la forêt domaniale du même nom, un bouquet d'arbres bien connu de la population locale sous le nom de " berceau du roi de Rome ). Les arbres en cause - chênes et charmes - forment un cercle de quelque huit mètres de diamètre. Au centre de ce cercle, deux chênes (l'un de $19 \mathrm{dcm}$ de tour) se font face: on reconnaît là le dispositif de plantation décrit en forêt de Boscodon. Voici le croquis que vient cle nous en faire M. le Chef de District CleRC:

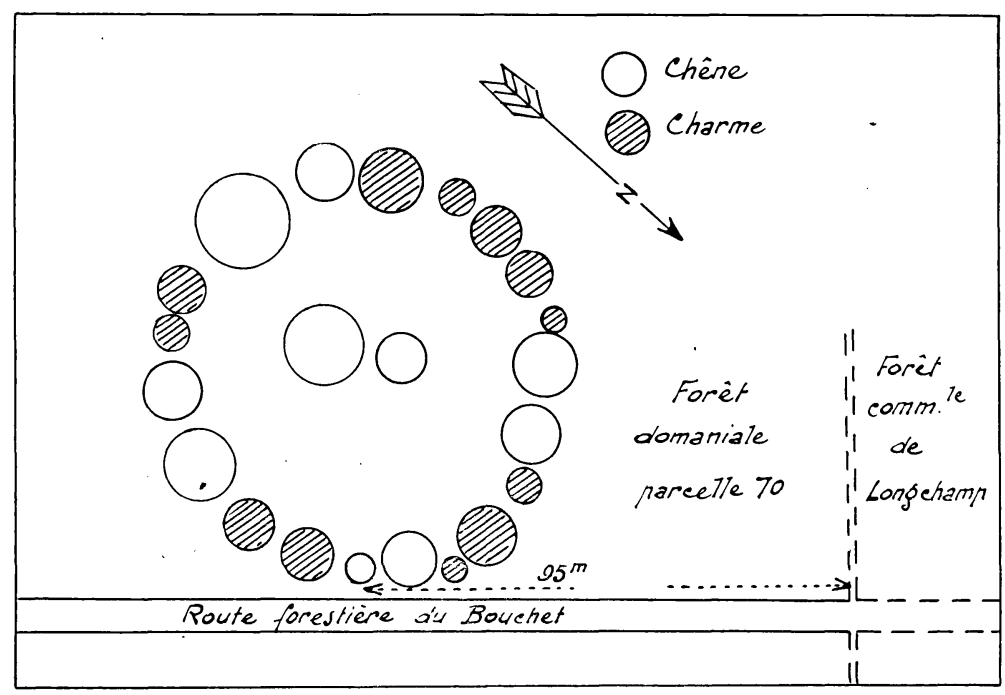

Le « Berceau du Roi de Rome », en forêt domaniale de Longchamp (Côte-d'Or) 
Le “ berceau du roi de Rome » est un lieu de promenade bien connu des habitants de la petite ville de Longchamp; on a toujours dit dans le pays - et on a raison - qu'il avait été planté pour fêter la naissance du fils de l'Empereur.

Souvenir sylvestre toujours vivant d'un prince malchanceux ! Demeure-t-il le dernier?

J. PARdé.

\section{Réimpression de deux Flores intéressantes}

Il est fréquent que des camarades forestiers nous demandent conseil dans le choix d'une «flore ». Mais choisir est une chose et se procurer en est une autre. Or, les deux ouvrages les plus utilisés en France étaient devenus introuvables, la Flore de Coste depuis un certain nombre d'années déjà, celle de Fournier plus récemment. Les éditions en étaient épuisées.

Nous sommes en mesure de signaler aux lecteurs de la Revue forestière française que ces deux Flores viennent d'être rééditées et il est possible de les retrouver en librairie. C'est une heureuse nouvelle pour tous ceux qui s'intéressent à la Botanique. Ces deux ouvrages figurent parmi leurs meilleurs outils de travail.

M. JaCAMON.

- Coste $(\mathrm{H})$

\section{Flore descriptive et illustrée de la France}

3 tomes, 416 pages +627 pages +807 pages.

Librairie des Sciences Naturelles. Paris (broché: environ $120 \mathrm{NF}$ ).

- Fournier (P.)

\section{Les quatre flores de France}

1 vol., 1105 pages. Editions Lechevalier. Paris (relié: 50 NF). 